\title{
MODELO MULTICRITÉRIO DE APOIO A DECISÃO PARA O PLANEJAMENTO DE MANUTENÇÃO PREVENTIVA UTILIZANDO PROMETHEE II EM SITUAÇÕES DE INCERTEZA
}

\author{
Cristiano Alexandre Virgínio Cavalcante* \\ Universidade Federal de Pernambuco (UFPE) \\ Recife - PE \\ cristiano@ufpe.br
}

\author{
Adiel Teixeira de Almeida \\ Universidade Federal de Pernambuco (UFPE) \\ Recife - PE \\ aalmeida@ufpe.br
}

* Corresponding author/autor para quem as correspondências devem ser encaminhadas

Recebido em 12/2004; aceito em 04/2005

Received December 2004; accepted April 2005

\begin{abstract}
Resumo
Ferramenta estratégica para redução dos custos e incremento da disponibilidade da planta, a Manutenção Preventiva, nos últimos anos, tem alcançado sua importância devida, com um crescimento significativo de sua aplicação nas indústrias. Apesar da existência de uma grande quantidade de modelos que tratam desta problemática, em muitas situações em que não se tem dados de falhas ou estes são pouco confiáveis e, além disso, deseja-se observar o comportamento de mais de um aspecto ao se estabelecer a periodicidade da manutenção preventiva, estes modelos fornecem resultados pouco satisfatórios. Neste contexto, este artigo traz um modelo de decisão que, fazendo uso do método multicritério PROMETHEE II e de análise Bayesiana, permite que se estabeleça a periodicidade da manutenção preventiva, observando-se o parâmetro custo e confiabilidade, além disso, permite que a ausência de dados não seja um empecilho para a estipulação destes tempos.
\end{abstract}

Palavras-chave: decisão multicritério; manutenção preventiva; políticas de manutenção.

\begin{abstract}
As a strategic tool to reduce costs and increment the availability of the plant, Preventive Maintenance has become quite popular over the last years due to the significant increase of its application in industries. Despite the great quantity of models that deal with that problem, these are less than satisfactory in many situations when failure data are inexistent or not too reliable and when the observation of the conduct of more than one aspect is desirable to establish the periodicity of Preventive Maintenance. In such context, this article offers a model of decision by making use of the multicriteria method PROMETHE II and the Bayesian analysis, which allows to establish the periodicity of the Preventive Maintenance, observing the costs and reliability parameter and also permitting the lack of data not to be an impediment in the stipulation of those timings.
\end{abstract}

Keywords: multicriteria decision; preventive maintenance; maintenance policies. 


\section{Introdução}

As ações de manutenção têm sofrido grandes mudanças nos últimos tempos. O aumento de complexidade e a grande inserção da automação, nos mais diversos sistemas de produção, enfatizam a grande importância da manutenção para os mais diversos sistemas, a fim de mantê-los em níveis de performance desejados, reduzir as paradas não planejadas e os altos custos provenientes destas.

A grande relevância da manutenção preventiva, aqui tomada como sendo o plano de substituição dos equipamentos, se dá pelo fato de que todos os equipamentos falham e estas falhas geram grandes conseqüências aos processos produtivos; conseqüências estas que nem sempre são de mesma gravidade. Em sistemas de produção de bens, as falhas significam: atraso de produção, retrabalho, ineficiência, desperdício de insumos, horas-extras, estoques altos, enfim, uma série de prejuízos que podem ser traduzidos em unidade financeira, através do custo que, na maioria das vezes, não atinge os clientes. Por outro lado, principalmente em sistemas de produção de serviços, a falha tem uma conotação bastante negativa e é de percepção imediata do cliente, quando não raro, afeta de forma direta, negativamente, àqueles que estão sendo atendidos. Neste sentido, a alta confiabilidade não é unicamente desejável; é essencial. Passa a ser um objetivo necessário que fornece a probabilidade que uma falha não ocorra, para um determinado instante e sobre condições predeterminadas. Contudo, seja nos sistemas de produção de bens e, principalmente, nos sistemas de produção de serviços, deseja-se antecipar às falhas, uma vez que os prejuízos advindos destas são diversos e muitas vezes difíceis de serem mensurados. Assim sendo, a observação da confiabilidade e o custo, juntos, permite um estabelecimento de uma política de manutenção preventiva mais estruturada e que mais facilmente alcança seu objetivo de preservação do sistema produtivo em estados específicos de performance.

Assim, este artigo faz ênfase aos sistemas de produção mais sensíveis a ocorrência de falhas e, neste domínio, trata o problema de planejamento de políticas de manutenção preventiva, sob o enfoque da metodologia de apoio multicritério a decisão, que se mostra bastante adequada no tratamento conjunto dos critérios custo e confiabilidade. Além disso, faz uso da metodologia bayesiana no procedimento para superar a ausência de dados de falhas.

\section{A Manutenção Preventiva}

As ações que, na tentativa de prevenir a ocorrência de falhas, são antecipadas através da substituição de partes do sistema constituem a manutenção preventiva, que na terminologia empregada neste trabalho refere-se ao plano de substituição de peças de equipamentos ou partes que podem falhar em operação, a menos que uma substituição seja feita a tempo. Neste contexto, a manutenção preventiva é apropriada para equipamentos cuja taxa de falhas cresce com o uso (Glasser, 1969; Barlow \& Proschan, 1965; Barlow \& Proschan, 1975).

Sendo assim, é considerado neste trabalho o modelo apresentado na Figura 1, em que: a taxa de falha dos equipamentos é crescente com o tempo e os equipamentos, tendo seus dispositivos substituídos, voltam ao estado de novo e suas falhas podem ser melhor representadas pelas seguintes distribuições: WEIBULL, NORMAL TRUNCADA e a GAMA, que caracterizamse por poderem representar o desgaste de um item (Almeida \& Souza, 2001).

Na construção de uma distribuição de falha, deve-se partir de qualquer evidência empírica na forma de dados históricos, julgamento ou qualquer combinação de ambos. Em algumas 
situações, pode-se construir uma distribuição de probabilidade diretamente a partir dos dados. Porém nem sempre é fácil se fazer associação dos dados com as informações necessárias para o planejamento de substituição, como também nem sempre os dados são suficientes para descrever a probabilidade de falha em vários intervalos de tempo com uma boa precisão. Uma abordagem alternativa para obtenção do modelo que descreve o comportamento das falhas de equipamentos, na dimensão do tempo, é assumir uma distribuição de falhas e então estimar os parâmetros da função assumida. A adoção de tal procedimento, muitas vezes, simplifica a análise matemática, na aplicação de um modelo de substituição, e traz vantagens na obtenção dos dados. Aqui, adota-se a distribuição Weibull que é útil em uma variedade de aplicações, particularmente, para o modelo da vida de dispositivos. Largamente usada, a Weibull assume uma variedade muito grande de formas e por isso é bastante flexível, podendo ser empregada para diversos tipos de dados (Nelson, 1982; Weibull, 1951).

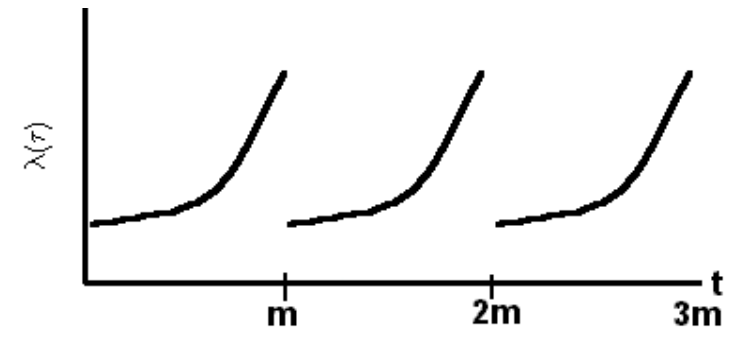

Figura 1 - Comportamento da taxa de falhas com a substituição $m_{i}$ realizadas em diferentes instantes.

A Weibull pode apresentar-se com dois parâmetros:

$\beta$ - parâmetro de forma;

$\eta$ - parâmetro de escala.

Funções importantes relacionadas com a variável aleatória tempo até a falha $(t)$ são mostradas logo abaixo:

Função densidade de probabilidade:

$$
\left.f(t)=(\beta / \eta)[t / \eta]^{\beta-1} e^{\left[-(t / \eta)^{\beta}\right.}\right]
$$

A função confiabilidade é dada por:

$$
R(t)=e^{\left[-(t / \eta)^{\beta}\right]}
$$

\section{Políticas de Substituição}

Os modelos de substituição são um conjunto de técnicas bastante importantes na manutenção preventiva, eles garantem uma diminuição dos custos referentes às substituições, procurando, de uma melhor forma, atender às necessidades de sistemas diferentes. Neste sentido, há diversos modelos propostos na literatura, os quais possuem características diferentes e são mais ou menos apropriados, a depender das circunstâncias e de fatores relacionados a: 
custos, desempenho funcional do equipamento e sua importância no processo produtivo (Jorgensen \& McCall, 1963).

A política de substituição por idade é um procedimento que consiste em substituir um item, no momento de sua falha ou ao atingir um tempo de vida $T$ (idade de substituição), seja qual for a situação que ocorra primeiro; por um adicional que será submetido às mesmas regras que o antigo. $\mathrm{O}$ uso dessa política só é eficaz se o custo de substituição antes que a falha se dê proporcione alguma economia em relação a substituição devido as falhas. A questão central, não só nesta política de substituição como nas outras, é qual idade faria uma unidade ser substituída com o menor custo por unidade de tempo de utilização?. Essa questão tem como resposta, na maioria das vezes, o resultado de problemas de otimização. Abaixo, segue a estrutura matemática para um modelo de substituição por idade com base no mínimo custo.

Há dois custos envolvidos:

$$
\begin{aligned}
& C a=\text { custo de substituição após a falha; } \\
& C b=\text { custo de substituição antes da falha. }
\end{aligned}
$$

Nesse caso, a hipótese utilizada é de que os intervalos entre substituições são suficientemente curtos para que se ignore o valor do dinheiro no tempo (Barlow, 1975; Glasser, 1969; Almeida \& Souza, 2001).

O custo esperado relativo à utilização de uma política de substituição por idade é dado pela expressão abaixo:

$$
C(t)=c_{a} \int_{0}^{t} f(x) d x+c_{b} \int_{t}^{\infty} f(x) d x
$$

Um outro conceito bastante importante é o período esperado de uso que expressa o tempo médio de uso de um determinado equipamento.

$$
T(t)=\int_{0}^{t} x f(x) d x+t \int_{t}^{\infty} f(x) d x
$$

Numa política de substituição por idade tem-se como principal objetivo garantir alta disponibilidade a um mínimo custo, através de sucessivas substituições que se antecedam às falhas. A noção de custo não faz sentido sem que esta esteja vinculada a dimensão de tempo. Sendo assim, a base para o estabelecimento da periodicidade das substituições fundamentase na obtenção de um período entre substituições que minimize a relação do custo esperado por unidade de tempo de utilização. Este parâmetro é representado, aqui, por $\mathrm{Cm}$ correspondendo a razão entre a expressão 3 e 4, sendo esta a função objetivo do problema de substituição ao mínimo custo, cuja a expressão analítica, segue abaixo:

$$
C m(t)=\left(c_{a} F(t)+c_{b}[1-F(t)]\right) /\left(\int_{0}^{t} x f(x) d x+t \int_{t}^{\infty} f(x) d x\right)
$$

\section{O Problema}

Como já se pode perceber, o problema principal no estabelecimento de uma política de manutenção preventiva repousa na escolha do tempo entre estas manutenções ou na frequiência com que elas são feitas. Contudo, o estabelecimento do tempo de substituição 
conduz a diferentes comportamentos dos aspectos mais importantes envolvidos com o funcionamento do equipamento, sejam estes: confiabilidade ou custos de manutenção e, não obstante, estes aspectos são conflitantes, o que reforça o caráter multicritério deste problema, onde os diferentes aspectos são os critérios, na linguagem desta metodologia; e os diferentes tempos são as alternativas, e, além disso, se deve levar em conta aspectos subjetivos do decisor para o estabelecimento de uma decisão mais adequada, tais como: sua preferência com relação a cada critério e a importância que cada critério tem sob seu julgamento.

Apesar da apropriação da metodologia multicritério no tratamento simultâneo de critérios conflitantes envolvidos no problema de substituição de equipamentos, na literatura, há poucas aplicações dessa metodologia na área de manutenção programada, mais raro, ainda, é o uso de uma abordagem multicritério de uma forma que permita o tratamento de incertezas relacionadas aos dados de manutenção. Por outro lado, há uma diversidade muito grande de textos que retratam a problemática de substituição de equipamentos, pelos mais diferentes enfoques e considerando aspectos bastante diferenciados, entretanto tais textos têm como característica, quase que geral, o uso do paradigma de otimização, onde um único critério ou função objetivo é considerado.

Neste sentido, antecipando as ferramentas utilizadas na construção do modelo na seção 7 , apresentam-se, a seguir, alguns trabalhos importantes dentro do contexto da manutenção programada sob a ótica de otimização, cuja fundamentação teórica permite o tratamento de incertezas, através de análise Bayesiana. Além disso, serão discutidos alguns artigos que fazem uso da abordagem de decisão multicritério, a fim de compreender seus mecanismos de tradução dos requisitos básicos da estrutura de um problema de substituição; ressaltar suas limitações e permitir identificar na modelagem multicritério pontos adequados para conexão com a abordagem Bayesiana, no tratamento de incertezas.

Dentro do contexto da manutenção programada, há alguns trabalhos que tratam a questão da incerteza fazendo uso da metodologia Bayesiana. Brint (2000) propõe um modelo que permite estender o intervalo entre as manutenções preventivas para os itens cujas falhas podem ser catastróficas. Makis \& Jardine (1992) propõem um modelo para a substituição ótima, que, de uma forma geral, tem como finalidade a especificação de regras simples de substituição que minimizam, a longo prazo, o custo médio esperado por unidade de tempo. Percy \& Kobbacy (1997) e (2000) seguem a mesma linha, contudo, também ressaltam as limitações e as hipóteses irrealistas associadas às diversas abordagens que tratam do planejamento da manutenção preventiva sem um cuidado maior da questão da incerteza nos dados de manutenção. Mauer \& Ott (1995) observam a importância e os efeitos de se considerar as incertezas quanto aos custos. Neste contexto, seguindo uma linha similar aos autores anteriores, apresentam um modelo, cujo objetivo é obter o valor de tempo ótimo entre substituições levando-se em consideração a incerteza quanto ao custo. Percy et al. (1997) consideram modelos estocásticos quando ainda há dados disponíveis, enfatizando a intervenção da manutenção preventiva, a fim de evitar a falha do sistema. Silver \& Fishter (1995) abordam o problema de periodicidade da manutenção sob o enfoque de análise Bayesiana combinada com procedimentos heurísticos.

Dentro do contexto da manutenção programada, há também, trabalhos que fazem uso da abordagem de apoio multicritério a decisão. Kralj \& Petrovic (1995) usam uma abordagem de otimização combinatória multiobjetivo. Gopalaswamy et al. (1993) propõem um modelo de decisão multicritério, onde três critérios são considerados: a taxa de custo de substituição mínima, a disponibilidade máxima e a confiabilidade do componente de base. Almeida (2005) apresenta um modelo baseado em teoria da utilidade multiatributo. Lotfi (1995) 
apresenta um modelo baseado em programação linear inteira mista multiobjetivo. Chareonsuk et al. (1997) fazendo uso da metodologia multicritério PROMETHEE, estabelecem o intervalo entre manutenções levando-se em consideração dois critérios importantes: o custo por unidade de tempo e a confiabilidade; contudo, os autores estabelecem faixas de valores para ambos os critérios, através deste procedimento, excluem as regiões de conflitos entre os critérios transformando o problema em um problema cuja solução sempre estará condicionada ao menor tempo dentre as alternativas.

\section{Apoio Multicritério a Decisão}

O apoio multicritério a decisão pode ser visto como um conjunto de métodos que se prestam a tornar claro um problema, no qual as alternativas são avaliadas por múltiplos critérios, os quais são conflitantes, na maioria dos casos. Neste contexto, freqüentemente faz-se uso de métodos que utilizam a abordagem de superação, a fim de selecionar um subconjunto de um conjunto finito de alternativas ou mesmo ordená-las. Há outras traduções para os métodos de superação, tais como: sobreclassificação, prevalência ou subordinação e síntese (Almeida \& Costa, 2003; Gomes et al., 2002).

De forma geral, os métodos de superação não admitem a compensação ilimitada de largas desvantagens e, além disso, levam em conta o fato que pequenas diferenças entre as avaliações das alternativas nem sempre são significantes (Vincke, 1992).

Considerado um elemento importante dentre os métodos baseados na relação de Sobreclassificação de valor (Keyser \& Peeters, 1994), contando com diversas e importantes aplicações, (Raju \& Kumar, 1999; Brans et al., 1998; Babic \& Plazibat, 1998) o PROMETHE é um método de fácil entendimento, de modo que os conceitos e parâmetros envolvidos em sua aplicação têm algum significado físico ou econômico de rápida assimilação pelo decisor. O método PROMETHEE (Preference Ranking Method for Enrichment Evaluation), consiste em construir e explorar uma relação de sobreclassificação de valores (Vincke, 1992; Brans \& Mareschal, 2002). Os métodos PROMETHEE foram propostos pela primeira vez em 1982 e desde então não cessaram de ser objeto de desenvolvimento e adaptações complementares (Brans \& Mareschal, 2002).

Eles são utilizados em problemas multicritério do tipo:

$$
\operatorname{Max}\left\{f_{l}(x), f_{2}(x), \ldots, f_{j}(x), \ldots, f_{k}(x) \quad \forall x \in A\right\}
$$

Onde $A$ é um conjunto finito enumerável de $n$ ações potenciais em $f_{j}(),. j=1,2, \ldots, k, k$ critérios, que são as aplicações de $A$ sobre o conjunto dos números Reais. Cada critério tem unidade própria e não tem restrições no caso em que certos critérios são para maximizar e outros são para minimizar.

A utilização do PROMETHEE exige o conhecimento de alguns conceitos utilizados em sua fase de construção da relação de sobreclassificação, tais conceitos são apresentados a seguir:

- $w_{j}$ é o peso de cada critério e significa a importância que o critério tem.

- $f_{j}(a)$ é o valor ou o desempenho da alternativa $a$ em relação ao critério $j$.

- $F_{j}(a, b)$ é a função de preferência, valor que varia de zero a um e representa o comportamento ou atitude do decisor frente às diferenças proveniente da comparação par a par entre as alternativas, para um dado critério. Comumente, são apresentadas seis formas mais utilizadas (Brans \& Mareschal, 2002). 
- $q$ representa um limite de indiferença, o maior valor para $\left[f_{j}(a)-f_{j}(b)\right]$ abaixo do qual existe uma indiferença.

- $\quad p$ representa o limite de preferência, o menor valor para $\left[f_{j}(a)-f_{j}(b)\right]$ acima do qual existe uma preferência estrita.

- $\Pi(a, b)$ é o grau de sobreclassificação de $a$ em relação a $b$, também é chamado de índice de preferência multicritério. É calculado por:

$$
\Pi(a, b)=\frac{1}{W} \sum_{j=1}^{n} w_{j} F_{j}(a, b) \quad \text { onde, } W=\sum_{j=1}^{n} w_{j}
$$

- $\Phi^{+}(a)$ é chamado de fluxo de saída e representa a média de todos os graus de sobreclassificação de $a$, com respeito às todas outras alternativas, é dado pela expressão:

$$
\Phi^{+}(a)=\sum_{b \in A} \frac{\Pi(a, b)}{n-1}
$$

Quanto maior $\Phi^{+}(a)$, melhor a alternativa.

- $\Phi^{-}(a)$ é chamado de fluxo de entrada, representa a média de todos os graus de sobreclassificação de todas as outras alternativas, sobre $a$, é dado pela expressão:

$\Phi^{-}(a)=\sum_{b \in A} \frac{\Pi(b, a)}{n-1}$

Quanto menor $\Phi^{-}(a)$, melhor é a alternativa.

O decisor deve, então, estabelecer para cada critério um peso $w_{j}$ que aumenta com a importância do critério. De acordo com o modo com que a preferência do decisor aumenta com a diferença entre o desempenho das alternativas para cada critério $\left[f_{j}(a)-f_{j}(b)\right]$, ele pode definir uma função $F_{j}(a, b)$ que assume valores entre zero e um. Estes valores aumentam se a diferença de desempenho, ou a vantagem de uma alternativa em relação à outra aumenta. E, são iguais a zero, se o desempenho de uma alternativa é igual ou inferior ao desempenho da outra.

Estabelecidas as intensidades de preferências, obtém-se o grau de sobreclassificação $\Pi(a, b)$ para cada par de alternativas $(a, b)$.

Em seguida, tem-se a fase de exploração das relações de sobreclassificação levantadas na fase de construção das mesmas. A fase de exploração tem como objetivo subsidiar o apoio a decisão, tendo em vista, as relações que foram construídas, as limitações do decisor e a problemática característica.

A família de métodos PROMETHEE se divide em:

- PROMETHEE I - Pré-ordem parcial, problemática de escolha;

- PROMETHEE II - Estabelece uma pré-ordem completa entre as alternativas, podendo ser utilizado também na problemática de escolha;

- PROMETHEE III - Ampliação da noção de indiferença, tratamento probabilístico dos fluxos (preferência intervalar);

- PROMETHEE IV - Pré-ordem completa ou parcial. Problemática de escolha e ordenamento. Destinado as situações em que o conjunto de soluções viáveis é contínuo; 
- PROMETHEE V - Nesta implementação, após estabelecer uma ordem completa entre as alternativas (PROMETHEE II), são introduzidas restrições, identificadas no problema, para as alternativas selecionadas; incorpora-se uma filosofia de otimização inteira;

- PROMETHEE VI - Pré-ordem completa ou parcial. Problemática de escolha e ordenamento. Destinado as situações em que o decisor não consegue estabelecer um valor fixo de peso para cada critério;

- PROMETHEE - GAIA - Extensão dos resultados do PROMETHEE, através de um procedimento visual e interativo.

\section{Abordagem Bayesiana}

A análise Bayesiana é apropriada quando não há dados o suficiente ou ausência completa de dados. Não sendo possível, nestas situações, a obtenção direta dos parâmetros da distribuição que, sobre a evidência de experiências anteriores, modelam bem estes tipos de dados. Desta forma, os parâmetros da distribuição Weibull são considerados variáveis aleatórias $\theta_{1}$ e $\theta_{2}$, cujas distribuições a priori do conhecimento do especialista sobre estas variáveis $\pi\left(\theta_{l}\right)$ e $\pi\left(\theta_{2}\right)$ devem ser eduzidas.

O modelo do conhecimento a priori de um especialista se baseia na hipótese de que um especialista tem uma idéia razoável, ou seja, uma expectativa sobre a distribuição de probabilidade subjetiva de uma variável $\theta$ representativa do estado da natureza, a qual pode ter um caráter aleatório ou não (Martz \& Waller, 1982).

\section{Estrutura do Modelo de Decisão}

O modelo de apoio multicritério a decisão, aqui construído, conserva alguns conceitos importantes dos modelos clássicos que tratam o problema de substituição de equipamentos (Jardine, 1992; Barlow \& Proschan, 1965). Tendo como base a política de substituição por idade, sob o paradigma do apoio multicritério a decisão e inspiração do modelo proposto por Chareonsuk et al. (1997), o modelo isola o parâmetro confiabilidade transformando-o num critério de escolha em adição ao critério custo unitário esperado, já usado nas abordagens clássicas. E, a fim de proporcionar a capacidade de tratar as incertezas relacionadas ao comportamento de falhas dos equipamentos em estudo, faz uso de elementos da escola Bayesiana.

Como Hipótese do modelo considera-se que:

- O conjunto de alternativa é discreto, ou seja, a manutenção é feita sob oportunidade, sendo assim, há um número finito de alternativas de tempos de substituição que correspondem a algumas datas ou valor de tempo decorrido cuja substituição far-se-ia oportuna;

- O equipamento está sujeito a desgaste, ou seja, tem taxa de falhas crescente;

- A substituição de um equipamento ou parte deste deixa o sistema com desempenho tão bom quanto novo;

- Os tempos de falha do equipamento podem ser modelados por uma distribuição de probabilidade;

- Os parâmetros da distribuição podem ser obtidos por meio de elicitação do conhecimento de especialista. 
A aplicação do modelo segue uma seqüência de etapas bem definidas:

Inicialmente um conjunto de alternativas é levantado, visando-se àqueles tempos oportunos para se fazer a substituição. Segue-se então a avaliação das alternativas nos critérios custo e confiabilidade, porém, devido a ausência dos dados, é necessário se fazer a elicitação das distribuições a priori do conhecimento do especialista sobre os parâmetros da distribuição dos tempos de falha do equipamento. Consideradas as incertezas na etapa anterior, parte-se para a etapa de decisão onde seguem todas as etapas de implantação do método PROMETHEE II que serão discutidas juntamente com as etapas precedentes logo a seguir.

\subsection{Estruturação do modelo multicritério}

A obtenção das alternativas é um procedimento bastante direto. Neste sentido, dá-se ênfase as etapas necessárias e que antecedem a implementação do método PROMETHEE, já que necessitam de um maior cuidado e constituem os aspectos chaves para o modelo, no que diz respeito à superação das incertezas e o entendimento do comportamento dos critérios. Assim, cada um dos critérios é estudado individualmente e as incertezas são consideradas através da inserção das distribuições a priori dos parâmetros da distribuição que modela os tempos de falha.

\subsubsection{Estudo do comportamento do custo esperado por unidade de tempo}

Representado por $C m$, é bastante utilizado em modelos de substituição, na maioria dos casos corresponde à função objetivo que deve ser otimizada, tendo a expressão 5 a sua forma mais comum (Glasser, 1969; Makis \& Jardine, 1992; Percy \& Kobbacy, 2000). No modelo aqui proposto ele é tratado como um dos critérios de escolha das alternativas de tempo tp. Para valores de tempos pequenos (freqüência de substituição alta) decorrem valores altos de $\mathrm{Cm}$, o que corresponde à intensa prática de manutenção preventiva, muitas vezes desnecessária. Por outro lado, para valores muito elevados de $t p$, o que corresponde à freqüência baixa de substituição, decorrem valores de $C m$, também relativamente altos, devido às falhas que não conseguiram ser evitadas, a tempo, pela substituição. Tal comportamento pode ser visto na Figura 2.

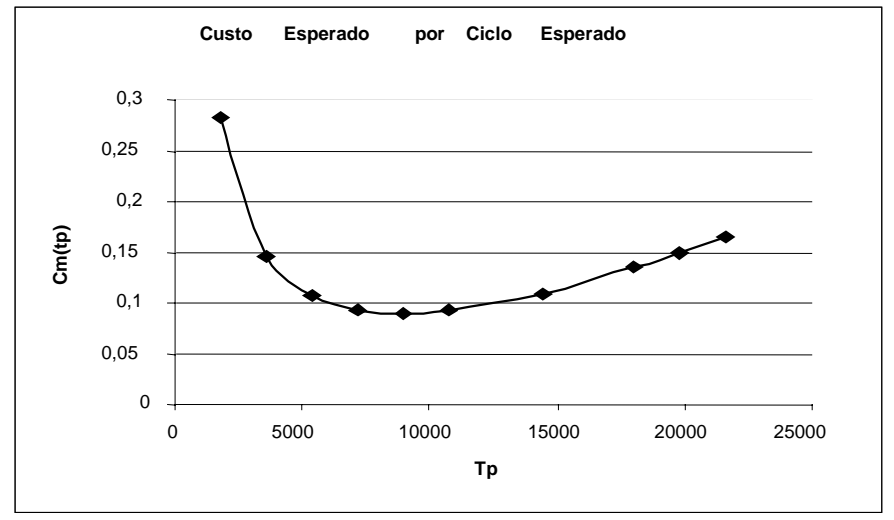

Figura 2 - comportamento do parâmetro $C m$ (razão do custo em unidade monetária pelo tempo em hora) com o Tp (tempo em hora). 
A curva que representa o $C m(t p)$ é tanto mais acentuada quanto maior for a razão entre os custos devido a uma falha e o custo de substituir antes que falhe $(\mathrm{Ca} / \mathrm{Cb})$, o que corresponde dizer que, para sistemas que apresentam grandes diferenças entre custos de falhar e substituir, mais evidentes são os benefícios provenientes de uma política de substituição.

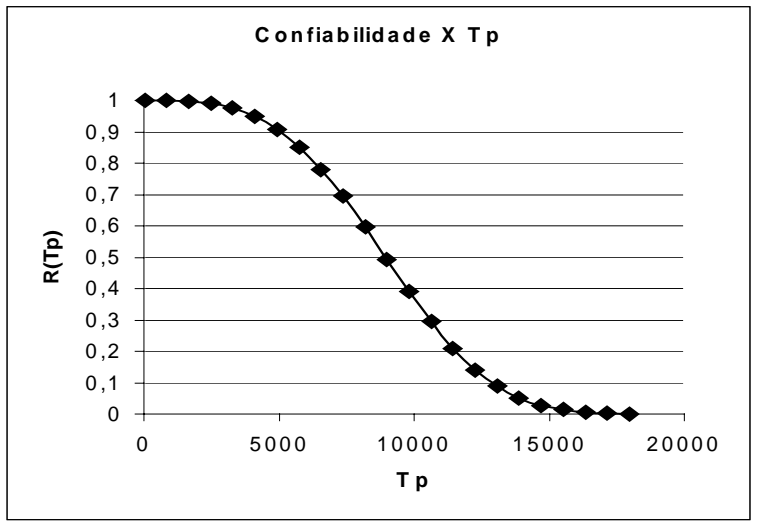

Figura 3 - Comportamento da confiabilidade com os tps dados em hora.

\subsubsection{Estudo do comportamento da confiabilidade $R(t p)$}

A confiabilidade tem o comportamento de uma função monotonicamente decrescente, ou seja, ao se prolongar os tempos entre substituição, a probabilidade de o equipamento falhar aumenta e quanto menor o tempo que um equipamento leva para ser substituído mais confiável ele está. Através da Figura 3, pode-se observar este comportamento.

\subsubsection{O conflito entre os critérios custo e confiabilidade}

O estudo dos critérios nos tópicos precedentes proporciona a identificação do comportamento bastante diverso dos critérios custo e confiabilidade. Na prática, no contexto da manutenção programada, deseja-se que o custo seja tanto menor quanto seja possível. Contudo, por outro lado, a confiabilidade tanto é melhor quanto maior for o seu valor, neste sentido, deseja-se maximizar este critério e minimizar o primeiro. Por tudo isso, dentro da perspectiva de otimização, considerando-se um dos critérios como restrição, o problema de substituição poderia ser considerado de duas diferentes formas, como segue:

$$
\text { Minimizar Cm(tp) }
$$

$\mathrm{Ou}$

$$
\text { Maximizar } R(t p)
$$

Porém, uma vez que se deseja considerar os dois critérios (funções objetivos), simultaneamente, e estes critérios são conflitantes, a noção de otimalidade não mais faz sentido. A seleção da melhor alternativa para os dois critérios obriga a um compromisso subjetivo entre os dois critérios, deixando de ser um processo puramente técnico (Clímaco et al., 2003). 


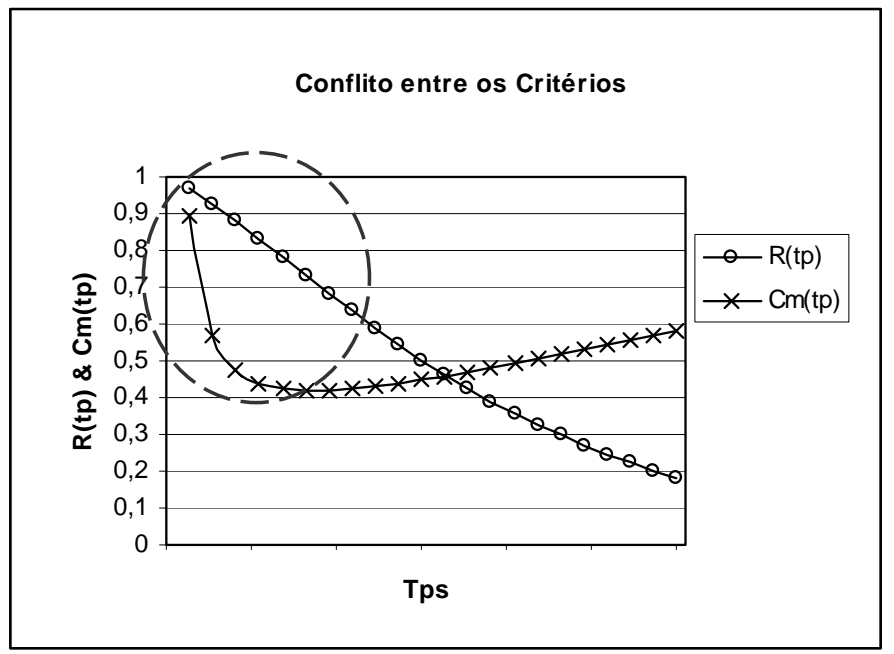

Figura 4 - Conflito entre os critérios custo e confiabilidade.

A Figura 4 permite a observação, simultânea, dos critérios custo e confiabilidade. Confrontando o comportamento dos dois critérios, torna-se evidente uma região de conflito entre os mesmos. Região essa em que as melhores alternativas de confiabilidade, correspondem as piores situações no critério custo e vice-versa, o que só reforça o forte conflito entre os critérios. Além disso, sob a perspectiva da análise de decisão, as alternativas que estão fora da região de conflito, em geral, tendem a ser dominadas (Vincke, 1992) pelas alternativas internas a essa, isto porque fora da região de conflito os critérios simultaneamente tendem a atribuírem piores avaliações as alternativas que lá estão. Neste sentido, na análise do problema de decisão, é de grande importância o estudo da região de conflito; estando nesta região as alternativas que estabelecem melhor trade-off entre os critérios.

\subsubsection{Tratamento de incertezas no modelo multicritério de apoio a decisão}

O estabelecimento de periodicidade das manutenções preventivas depende do comportamento relativo às falhas do item em estudo, contudo, é muito comum a ausência dos dados necessários para levantar a distribuição que modela o comportamento das falhas (Evans, 1991). Assim, a análise bayesiana é bastante útil ao estabelecer a possibilidade de considerar diversos tipos de dados (Percy et al., 1997; Mauer \& Ott, 1995; Silver \& Fishter, 1995).

Considerando-se a distribuição Weibull como sendo o modelo de falhas. No caso de ausência de dados e conseqüente incerteza em ambos os parâmetros dessa distribuição, pode-se recorrer ao conhecimento de especialistas e, sob a ótica da probabilidade subjetiva, os parâmetros da distribuição Weibull são considerados variáveis aleatórias $\theta_{1}$ e $\theta_{2}$, cujas distribuições a priori do conhecimento do especialista sobre estas variáveis $\pi\left(\theta_{1}\right)$ e $\pi\left(\theta_{2}\right)$ devem ser levantadas, a partir de um processo de elicitação (Raiffa, 1970).

Neste sentido, as expressões 9 e 10 correspondentes aos critérios (funções objetivo) custo e confiabilidade respectivamente, a fim de considerarem a incerteza sobre os parâmetros $\eta$ e $\beta$, os quais são modelados pelas distribuições $\pi(\eta)$ e $\pi(\beta)$, tomam, respectivamente, a forma das expressões 11 e 12 . 


$$
\begin{aligned}
& \operatorname{Min} \frac{c_{a} \int_{0}^{t} \int_{-\infty}^{+\infty} \int_{-\infty}^{+\infty} \pi(\beta) \pi(\eta) f(x) d x d \eta d \beta+c_{b} \int_{t}^{\infty} \int_{-\infty}^{+\infty} \int_{-\infty}^{+\infty} \pi(\beta) \pi(\eta) f(x) d x d \eta d \beta}{\int_{0}^{+\infty} \int_{-\infty}^{+\infty} \pi(\beta) \pi(\eta) x f(x) d x d \eta d \beta+t \int_{t}^{+\infty} \int_{-\infty}^{+\infty} \int_{-\infty}^{+\infty} \pi(\beta) \pi(\eta) f(x) d x d \eta d \beta} \\
& \operatorname{Max} 1-\int_{-\infty}^{t} \int_{-\infty}^{+\infty} \int_{-\infty}^{+\infty} \pi(\beta) \pi(\eta) f(x) d x d \eta d \beta
\end{aligned}
$$

\subsubsection{Agregação dos critérios}

Tratadas as incertezas, as alternativas recebem avaliações quanto ao custo de manutenção por unidade de tempo $(\mathrm{Cm})$ e quanto ao critério confiabilidade $(R)$, estas avaliações apresentadas na forma matricial dão origem ao que comumente se chama de matriz de desempenho dos critérios $\left\|f_{i j}\right\|$, que corresponde à avaliação da alternativa $i$ sobre o critério $j$.

Tabela 1 - Desempenho das alternativas.

\begin{tabular}{|c|c|c|}
\hline & $R$ & $C m$ \\
\hline$A_{1}$ & $R\left(a_{1}\right)$ & $C m\left(a_{1}\right)$ \\
\hline$A_{2}$ & $R\left(a_{2}\right)$ & $C m\left(a_{2}\right)$ \\
\hline$:$ & $:$ & $:$ \\
\hline$A_{i}$ & $R\left(a_{i}\right)$ & $C m\left(a_{i}\right)$ \\
\hline$:$ & $:$ & $:$ \\
\hline$A_{n}$ & $R\left(a_{n}\right)$ & $C m(n a)$ \\
\hline
\end{tabular}

Em geral, os dados da Tabela 1 não induzem a um ranking das alternativas de $A$ e nenhuma alternativa é ótima em todos os critérios simultaneamente, devido aos conflitos existentes entre os critérios. Sendo assim, um meio bastante adequado, neste tipo de situação, é o uso da abordagem de apóio a decisão multicritério (Brans \& Mareschal, 2002).

Como largamente já discutido, anteriormente, o método PROMETHEE, apesar de não se tratar de uma teoria fundamentada em axiomas, foi escolhido devido à apropriação de suas características ao problema, sua facilidade de compreensão e a forma com que avalia as alternativas: através de comparações relativas, onde uma alternativa não pode ser julgada boa por si só, pois é necessário que seja observado o desempenho desta alternativa relativo ao desempenho das outras.

Sendo assim, como se dá em muitos processos de julgamento de valor pessoal, na vida real, o decisor observando duas alternativas $(a, b)$, visualiza a diferença entre elas $d_{j}(a, b)$ com respeito aos desempenhos respectivos para um dado critério $j$, e então, afirma sob seu ponto de vista, representado por uma função chamada de critério generalizado $F_{j}\left(d_{j}(a, b)\right)$, se uma alternativa é melhor $\left(a P_{j} b\right)$, é tão boa quanto $\left(a I_{j} b\right)$ ou é pior $\left(b P_{j} a\right)$ ou se não podem ser comparadas $\left(a R_{j}{ }^{\prime} b\right)$. Estendendo este raciocínio para um conjunto com várias alternativas, sob a perspectiva de mais de um critério, as mesmas relações são validas, porém, são construídas sob o âmbito global, em que cada alternativa é avaliada com todas as outras do conjunto em todos os critérios. 
Em seguida, tem-se a fase de exploração das relações de sobreclassificação que foram levantadas na fase de construção. A fase de exploração tem como objetivo subsidiar o apoio a decisão, tendo em vista, as relações que foram construídas, os objetivos e limitações do decisor, e a problemática característica. Nesta etapa, levando-se em consideração os aspectos supracitados, o método PROMETHEE é definido e aplicado, podendo, seu resultado ser uma pré-ordem completa entre as alternativas, uma pré-ordem parcial ou uma sofisticação dessas duas últimas.

\section{Aplicação Numérica}

Baseado em um emprego do modelo feito no contexto de uma empresa de distribuição de energia, faz-se uma aplicação numérica que é formulada com valores próximos da realidade. Neste sentido, um equipamento foi selecionado, com o intuito de se planejar as suas manutenções preventivas. Diante das características do equipamento, foi lhe atribuída, como mais apropriada, a política de substituição por idade, assim, a questão que se quer responder, através da aplicação do modelo, é qual o tempo mais apropriado entre substituições? Além disso, na aplicação desta política, faz-se necessário, o conhecimento dos tempos de falha e os custos de substituir $(\mathrm{Cb})$ e reparar $(\mathrm{Ca})$.

Tabela 2 - Dados de entrada.

\begin{tabular}{|c|c|c|}
\hline \multicolumn{3}{|c|}{ Características do Equipamento } \\
\hline \multirow{2}{*}{ Weibull } & $\beta$ & Desconhecido \\
\cline { 2 - 3 } & $\eta$ & Desconhecido \\
\hline \multirow{2}{*}{ Custos } & Custo de Subs Cb & 400 \\
\cline { 2 - 3 } & Custo de Falha Ca & 2200 \\
\hline
\end{tabular}

Seguindo-se os passos do modelo de decisão, uma vez selecionado o equipamento, é necessário levantar o conjunto das alternativas do problema de decisão que correspondem aos valores de tempos mais oportunos para que as substituições sejam feitas. Na Tabela 3, apresenta-se o conjunto de ações $T i$ em duas diferentes unidades.

Tabela 3 - Conjunto de ações.

\begin{tabular}{|c|c|c|}
\hline Alternativas & Tempo em hora & Tempo em mês \\
\hline T1 & 720 & 1 \\
\hline T2 & 1440 & 2 \\
\hline T3 & 2160 & 3 \\
\hline T4 & 2880 & 4 \\
\hline T5 & 3600 & 5 \\
\hline T6 & 4320 & 6 \\
\hline T7 & 5760 & 8 \\
\hline T8 & 7200 & 10 \\
\hline T9 & 7920 & 11 \\
\hline T10 & 8640 & 12 \\
\hline
\end{tabular}


Em geral, o passo seguinte ao estabelecimento das alternativas nos modelos de decisão é a construção da matriz de avaliação dos critérios. Porém, devido à existência de incertezas relativas aos parâmetros da distribuição dos tempos de falha, antes de se calcular o desempenho de cada alternativa em cada critério, deve-se fazer o processo de elicitação para os parâmetros de incerteza, fazendo uso da já apresentada análise bayesiana, a fím de se obter as distribuições a priori de cada um dos parâmetros desconhecidos.

Assim feito, apresentam-se, inicialmente, os parâmetros da distribuição que modela a variabilidade do parâmetro $\eta$, essa distribuição simula a distribuição a priori do conhecimento do especialista sob o parâmetro de incerteza, caso esta tivesse sido obtida por um processo de elicitação. Neste sentido, conservando valores dentro da realidade, pode-se observar os parâmetros de $\pi(\eta)$ expostos na Tabela 4 . Também como pode-se observar o formato das distribuições a priori e acumulada na Figura 5.

Tabela 4 - Parâmetros da distribuição Weibull para o $\eta$ desconhecido.

\begin{tabular}{|c|c|}
\hline \multicolumn{2}{|c|}{ Parâmetros da Função Weibull } \\
\hline$\beta$ & 2,015 \\
\hline$\eta$ & 4683,45 \\
\hline Coef.cor & 0,981741 \\
\hline
\end{tabular}

De forma similar aos procedimentos adotados anteriormente para o parâmetro $\eta$, obtém-se a distribuição do parâmetro da Weibull para os valores de $\beta$. Ver Tabela 5.

Obtidas as distribuições a priori $\pi(\eta)$ e $\pi(\beta)$. Pode-se então seguir a construção da matriz de avaliação dos critérios custo e confiabilidade, cujos valores, para cada alternativa, são obtidos através das expressões 11 e 12 respectivamente. Sendo exibidos os valores para cada alternativa na Tabela 6.

Levantada a Matriz de avaliação dos critérios, segue-se o processo de agregação, onde deseja-se julgar as alternativas com respeito a ambos os critérios, levando-se em consideração os aspectos de preferência do decisor.
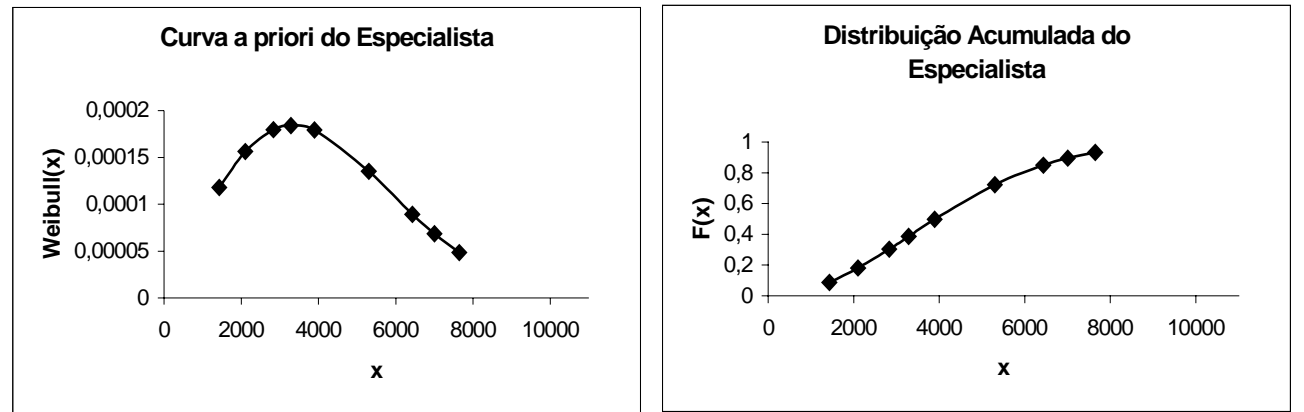

Figura 5 - Curva de distribuição a priori sob o conhecimento do especialista. 
Neste contexto, através de um processo interativo entre o decisor e o analista de decisão, determina-se a função de critério generalizado $\left(F_{j}().\right)$ que melhor modela o comportamento do decisor diante das amplitudes das diferenças $\left(d_{j}().\right)$ entre as avaliações para cada critério $\left(f_{j}().\right)$. Obtendo-se, com isso, os patamares de indiferença e de preferência. Na Tabela 7 são mostrados o tipo das funções de critério generalizado e os seus respectivos parâmetros.

Tabela 5 - Parâmetros da distribuição a priori do $\beta$.

\begin{tabular}{|c|c|}
\hline \multicolumn{2}{|c|}{ Parâmetros da Função Weibull } \\
\hline$\beta$ & 3,2 \\
\hline$\eta$ & 2,5 \\
\hline
\end{tabular}

A partir de então, obtidas as intensidades de preferência entre os pares de ações, comparadas, para cada critério, separadamente, pode-se, então, estabelecer o índice de preferência multicritério $\Pi(a, b)$, que representa uma medida de preferência de $a$ sobre $b$, levando-se em consideração todos os critérios. Todavia, para o cálculo dos $\Pi(a, b)$, o decisor deverá refletir sobre a importância que cada critério tem, estabelecendo pesos a cada um deles. Quanto maior a importância de um critério $j$, aos olhos do decisor, maior é o peso $w_{j}$ associado a ele, o que amplifica sua contribuição no cálculo de $\Pi(a, b)$. Dando sequiência nas etapas prescritas no método PROMETHEE, o próximo passo é o cálculo dos fluxos de entrada e de saída de cada alternativa, os quais permitem o estabelecimento das relações de sobreclassificação.

Tabela 6 - Matriz de avaliação do problema de decisão.

\begin{tabular}{|c|c|c|c|}
\hline Alternativas & $\mathrm{Tp}(\mathrm{h})$ & $\mathrm{R}(\mathrm{tp})$ & $\mathrm{Cm}(\mathrm{tp})$ \\
\hline $\mathrm{T} 1$ & 720 & 0,90497 & 0,82261 \\
\hline $\mathrm{T} 2$ & 1440 & 0,77434 & 0,61947 \\
\hline $\mathrm{T} 3$ & 2160 & 0,64003 & 0,57873 \\
\hline $\mathrm{T} 4$ & 2880 & 0,51658 & 0,57048 \\
\hline $\mathrm{T} 5$ & 3600 & 0,40983 & 0,57148 \\
\hline T6 & 4320 & 0,32124 & 0,57492 \\
\hline $\mathrm{T} 7$ & 5760 & 0,19253 & 0,58214 \\
\hline T8 & 7200 & 0,11397 & 0,58685 \\
\hline T9 & 7920 & 0,08777 & 0,58818 \\
\hline T10 & 8640 & 0,06789 & 0,58909 \\
\hline
\end{tabular}

Tabela 7 - Função de preferência e características dos critérios.

\begin{tabular}{|c|c|c|}
\hline Características & $R$ & $\mathrm{Cm}$ \\
\hline Max/Min & Max & Min \\
\hline Peso & 0,4 & 0,6 \\
\hline Função preferência & Tipo V & Tipo V \\
\hline Limiar de indiferença & 0,02 & 0,04 \\
\hline Limiar de preferência & 0,1 & 0,15 \\
\hline
\end{tabular}


Tabela 8 - Ordenamento das alternativas.

\begin{tabular}{|c|c|c|c|c|}
\hline Alternativas & $\mathrm{Tp}(\mathrm{h})$ & $\phi^{+}$ & $\phi^{-}$ & $\phi^{+}-\phi^{-}$ \\
\hline T3 & 2160 & 0,404 & 0,089 & 0,315 \\
\hline T4 & 2880 & 0,386 & 0,133 & 0,253 \\
\hline T2 & 1440 & 0,422 & 0,239 & 0,183 \\
\hline T5 & 3600 & 0,327 & 0,178 & 0,150 \\
\hline T6 & 4320 & 0,281 & 0,212 & 0,069 \\
\hline T7 & 5760 & 0,204 & 0,269 & $-0,065$ \\
\hline T1 & 720 & 0,400 & 0,600 & $-0,200$ \\
\hline T8 & 7200 & 0,086 & 0,303 & $-0,218$ \\
\hline T9 & 7920 & 0,084 & 0,326 & $-0,241$ \\
\hline T10 & 8640 & 0,084 & 0,328 & $-0,245$ \\
\hline
\end{tabular}

Faz-se, então, uso do método PROMETHEE II, o qual, através da diferença entre os fluxos positivo e negativo, estabelece uma pré-ordem completa entre as alternativas avaliadas. Assim, o que se estabelece como resultado da aplicação do Modelo Multicritério de Apoio a Decisão é o ranking das alternativas, estando ordenadas da melhor a pior. Ver Tabela 8.

A melhor alternativa, que ocupa o primeira lugar no ranking, é a alternativa $T 3$ e corresponde a realização da substituição do equipamento estudado a cada 2.160 horas de funcionamento ou um intervalo de 3 meses.

Obtidos os resultados da etapa anterior, foram feitas variações nos parâmetros do modelo, cujo o comportamento se mostrou robusto, não havendo alteração dos resultados para um intervalo considerável de variação.

\section{Conclusões}

Neste artigo foi proposto um modelo multicritério de apoio a decisão para o planejamento de manutenção preventiva que é capaz de contornar as duas principais dificuldades relacionadas com tal problema, quais sejam: poder decidir qual a periodicidade das substituições para um determinado item com base em mais de um critério e poder, mesmo quando não houver dados de falhas, prover um apoio adequado ao decisor na difícil tarefa de decidir o tempo mais oportuno para a substituição de um item.

Além disso, em relação a temática proposta, a manutenção preventiva mereceu investigação mais precisa, para denotar suas aplicações, acerca das políticas de substituição e os problemas que carregam. Sob a forma de pesquisa bibliográfica, puderam ser observados importantes trabalhos, os quais, nos mais diferentes enfoques, trataram da substituição de equipamentos.

Por fim, sob o contexto prático, a proposição do modelo suporta uma das grandes preocupações das estruturas de manutenção em organizações que lidam com vultuosas quantias imobilizadas em ativos físicos de suas plantas de produção.

\section{Agradecimentos}

Este trabalho foi apoiado parcialmente pelo CNPq. 


\section{Referências Bibliográficas}

(1) Almeida, A.T. de \& Souza, F.C.M. (2001). Gestão da manutenção na direção da Competitividade. Recife, Editora Universitária.

(2) Almeida, A.T. de \& Costa, A.P.C.S. (2003). Aplicações com Métodos Multicritério de Apoio a Decisão. Editora Universitária.

(3) Almeida, A.T. de (2005). Modelagem Multicritério para Seleção de Intervalos de Manutenção Preventiva Baseada na Teoria da Utilidade Multiatributo. Pesquisa Operacional, 25(1), 69-81.

(4) Barlow, R.E. \& Proschan, F. (1965). Mathematical Theory of Reliability. John Wiley \& Sons.

(5) Barlow, R.E. \& Proschan, F. (1975). Reliability and Life Testing Probability Models. Holt, Rinehart and Winston.

(6) Brans, J.P. \& Mareschal, B. (2002). Promethee-Gaia, une Methodologie d'Aide à la Décision em Présence de Critères Multiples. Éditions Ellipses, Bruxelles.

(7) Brint, A.T. (2000). Sequential Inspection Sampling To Avoid Failure Critical Items Being In An At Risk Condition. The Journal of the Operational Research Society, 51(9), 1051-1059.

(8) Chareonsuk, C.; Nagarura, N. \& Tabucanona, M.T. (1997). A multicriteria approach to the selection of preventive maintenance intervals. International Journal of Production Economics, 49(1), 55-64.

(9) Clímaco, N.C.; Antunes, C.H. \& Alves, M.J.G. (2003). Programação Linear Multiobjetivo: Do Modelo de Programação Linear Clássico à Consideração Explícita de Várias Funções Objetivo. Universidade de Coimbra.

(10) Dekker, R. (1996). Application of Maintenance optimization models: review and analysis. Reliability Engineering and System Safety, 51, 229-240.

(11) Evans, R.A. (1991). O Data, Data! Wherefore Art Than Data? IEEE Trans. Reliability, 40(5), 497.

(12) Glasser, G.J. (1969). Planned Replacement: Some Theory and its Application. Journal of Quality Technology, 1(2), 110-119.

(13) Goldman, A.S. \& Slattery, T.B. (1977). Maintainability: A Major Element of System Effectiveness. Robert E. Krieger, New York.

(14) Gomes, L.F.A.M.; Gomes, C.F.S. \& Almeida, A.T. (2002). Tomada de Decisão Gerencial: Enfoque Multicritério. Editora Atlas, Rio de Janeiro.

(15) Gopalaswamy, V.; Rice, J.A. \& Miller, F.G. (1993). Transit Vehicle Component Maintenance Policy Via Multiple Criteria Decision Making Methods. The Journal of the Operational Research Society, 44(1), 37-50.

(16) Jorgensen, D.W. \& McCall, J.J. (1963). Optimal Replacement Policies for a Ballistic Missile. Management Science, 9(3), 358-379.

(17) Kobbacy, K.A.H.; Percy, D.F. \& Fawzi, B.B. (1997). Small Data Sets And Preventive Maintenance Modeling. Journal of Quality in Maintenance, 3(2), 136-142. 
(18) Kralj, B. \& Petrovic, R. (1995). A Multiobjective Optimization Approach To Thermal Generating Units Maintenance Scheduling. European Journal of Operational Research, 84(2), 481-493.

(19) Lotfi, V. (1995). Implementing Flexible Automation: A Multiple Criteria Decision Making Approach. International Journal of Production Economics, 38(2-3), 255-268.

(20) Makis, V. \& Jardine, A.K.S. (1992). Optimal Replacement in the Proportional Hazards Model. INFOR, 30(2), 172.

(21) Martz, H.F. \& Waller, R.A. (1982). Bayesian Reliability Analysis. John Wiley \& Sons.

(22) Mauer, D.C. \& OTT, S.H. (1995). Investment Under Uncertainty: The Case Of Replacement Investment Decisions. Journal of Financial and Quantitative Analysis, 30(4), 581-605.

(23) Nelson, W. (1982). Applied Life Data Analysis. Wiley \& Sons.

(24) Percy, D.F. \& Kobbacy, K.A.H. (2000). Determining Economical Maintenance Intervals. International Journal of Production Economics, 67(1), 87-94.

(25) Percy, D.F.; Kobbacy, K.A.H. \& Fawzi, B.B. (1997). Setting Preventive Maintenance Schedules When Data Are Sparse. International Journal of Production Economics, 51(3), 223-234.

(26) Raiffa, H. (1970). Decision Analysis. Addison-Wesley.

(27) Silver, E.A. \& Fiechter, C.-N. (1995). Preventive Maintenance With Limited Historical Data. European Journal of Operational Research, 86(1), 125-144.

(28) Vincke, P. (1992). Multicriteria decision-aid. John Wiley \& Sons, Bruxelles.

(29) Weibull, W. (1951). A Statistical Distribution Function of Wide Applicability. Journal of Applied Mechanics, 18, 293-297. 